\title{
Case report of Odontogenic Sinusitis with multiple complications - case study
}

\author{
Dr. Kr. Bozov', Dr. Iv. Hristov', Dr. R. Strashimirova', M. Milkov² \\ 'ENT department, „MHAT Shumen“ \\ ${ }^{2}$ Department of Prosthetic Dentistry, Faculty of Dental Medicine, Medical University Varna
}

Summary:

A case of pansinuitis has been described after a tooth extraction, complicated by orbital cellulitis, peritonsillar abscess and cavernous sinus thrombosis.
Clinical symptoms, diagnostic problems, therapeutic approach and the results of the treatment are unveiled below.

We present a clinical case as the only case in our long-standing practice and, even more, in the available scientific literature.

A description of such a case was found only in „ENT Journal“" by N. A. Zabelin, where a 17-year old patient from Leningrad in 1988 was described with peritonsillar abscess, purulent pansinusitis and a subperiostal orbital abscess, who subsequently developed tonsillogenic sepsis.

\section{Clinical Presentation}

We present a case of a 22-year old woman, who sought emergency medical attendance for a history of severe sore throat, headache, swelling and redness of the right eye with diplopia and fever up to $39^{\circ} \mathrm{C}$ (fig. 1) The patient reported a tooth extraction on the right side in the recent weeks prior to the dramatic condition. Throughout the interrogation she denied any significant past diseases, family history or exposure to risk factors.

By the time of our examination we encountered severely hyperaemic and swollen mucosa of the right nasal passage with thick purulent secretion in the middle nasal meatus. The pharyngeal mucosa was hyperaemic with manifest swelling of the right peritonsilar area, also affecting the uvula. Otoscopy revealed that both ears were intact. 


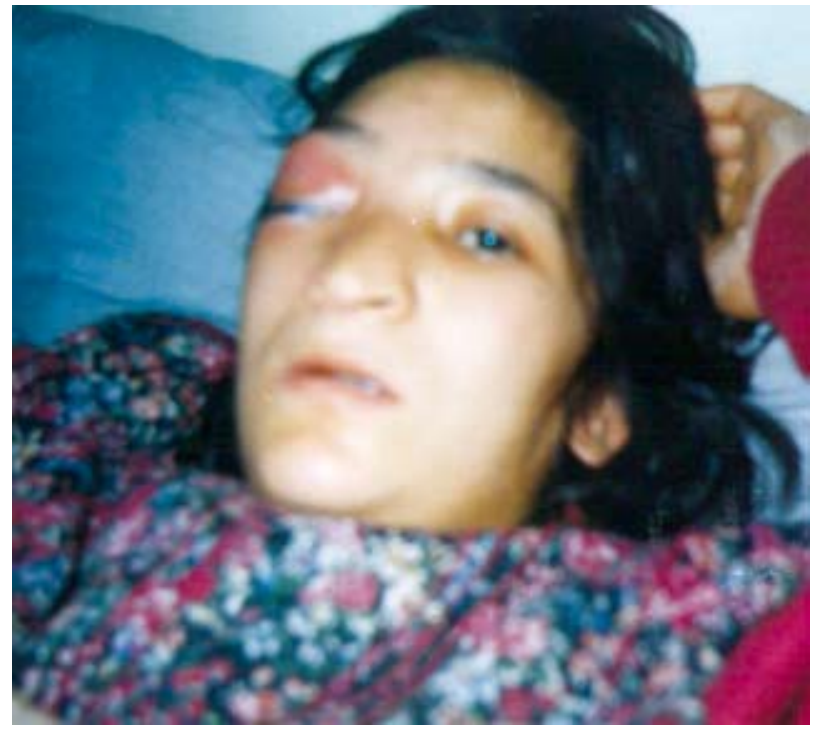

Fig. 1. The female patient, 22, presenting with exophthalmos and swollen eyelids

Later, we conducted ophthalmology examination which showed oedema of the eyelids, especially on the right side with impaired movements of the right eyeball in all directions. The right pupil was wide without direct light reaction; the papilla appeared normal. The neurologic status was normal and the lumbar puncture brought clear liquor with normal pressure.

The laboratory findings were as following:

ERS - 80 mm/h; WBC - 19,2 G/L; Neu\% - 97,4\%; LYM - 2,42 G/L; Coagulogram: PA - 85\%; Fibrinogen $-8,1 \mathrm{G} / \mathrm{L}$; Urine: protein (+). Microbiology of the peritonsillar abscess discharge detected Str. Anginosus, with normal susceptibility.

\section{Radiology findings}

$\mathrm{X}$-ray of the thorax - normal imaging.

The CT scan of the head showed almost entire opacification of the right maxillary and ethmoid sinus with gas-fluid levels in the right maxillary sinus. Moreover, opacification of the anterior and lateral walls of the right orbit were seen. The brain showed normal imaging, no focal infections or oedema were localized. (fig. 2)
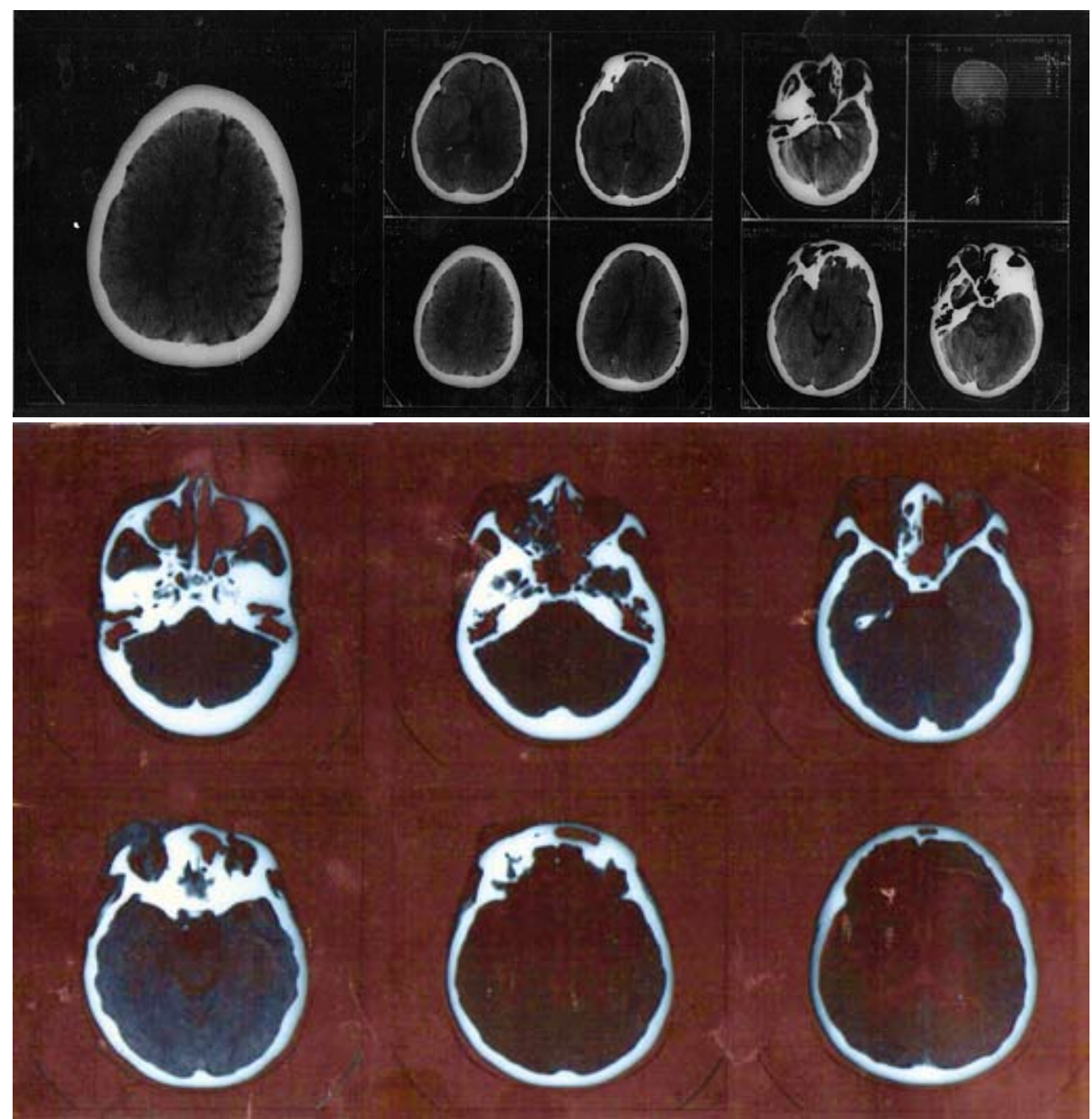

Fig. 2. CT scan of the head - axial view 


\section{Surgical procedure and outcome}

The patient was admitted to the intensive care facility after a consult with an anaesthesiologist due to the rapid worsening of her condition. Several manipulations were performed in the first days of her treatment. First, the peritonsillar abscess was drained and gross amount of purulent malodorous exudate was evacuated. Then, a puncture of the right maxillary sinus led to the evacuation of malodorous purulent discharge from the cavity. On the second day the exophthalmos and the swelling of the eyelids were even more prominent. The local status of the patient was poorly affected by the antibiotic treatment and purulent discharge continued to be evacuated on the consequent revisions of the abscess and the sinus cavity. When the general condition of the patient was finally stabilized, she was transferred to the ENT ward.

Due to the worsening of her condition and considering the laboratory and CT findings, mentioned above, on the sixth day of her treatment we decided to perform radical surgery. Under general anaesthesia, we made a wide incision beneath the right eyebrow and thick purulent exudate was drained. Then, we performed a radical antrostomy with a communication to the inferior nasal meatus where we removed thickened mucosa with necrotic, soft areas and foul-smelling purulent discharge.

Therefore, the postoperative period was clear. The purulent discharge withdrew and the swelling of the eyelids quickly disappeared. The movement of the eyeball was completely recovered (visus $1,0)$. The laboratory markers returned to normal (ERS $6 \mathrm{~mm} / \mathrm{h}$; WBC $-6,8 \mathrm{G} / \mathrm{L}$ ). The secondary CT scan showed the paranasal sinus were free of fluid. Eventually, the patient was dismissed of the hospital on the 15th day in radically improved local and general condition.

\section{Differential diagnosis}

1. Initiating Cavernous sinus thrombosis

2. Retrobulbar haematoma

3. Orbital tumor.

\section{Therapeutic regimen}

Day 1 - Day 9:

- Antibiotics:

- Amikacin 2x500mg;

- Metronidazole 2x250mg;

- Cefepime 2x2,0g;

- Diclofenac lamp i.m.

- Metamizole 3x2ml amp

- Enoxaparin 40mgs.c

- Ascorbic acid 2x5ml;

- Intravenous fluids: Mannitol 10\% 2x250ml; $5 \%$ sugar solution $+8 \mathrm{E}$ insulin. $+\mathrm{KCl} 15 \%$ $10 \mathrm{ml} .+\mathrm{NaCl} 10 \% 30 \mathrm{ml}$.

- Xylometazoline $0,1 \%$ locally $5 x$ daily

- Day 9 - Day 15

- Penicillin 4 x 5mln.E

- Amikacin 2 x 500

- Methylprednisolone x 20mg

- Gentamycin Ophthalmic ung.

\section{Conclusion}

Knowledge, early diagnosis and appropriate treatment of complicated odontogenic sinusitis are of considerable importance, as such infections can end with severe consequences for the patient.

In addition to the clinical picture, imaging is a key to accurate diagnosis prior to planning a proper surgical approach.

\section{References}

1. Arias-Irimia O, Barona-Dorado C, Santos-Marino JA, Martínez-Rodriguez N, Martínez-González JM. Meta-analysis of the etiology of odontogenic maxillary sinusitis. Med Oral Patol Oral Cir Bucal. 2010; 15(1): e70-3.

2. Anderson R. Diodrast studies of the vertebral and cranial venous systems to show their probable role in cerebral metastases. J Neurosurg. 1951; 8(4): 411-22.

3. Gwaltney JM Jr. Acute community-acquired sinusitis. Clin Infect Dis. 1996; 23(6): 1209-23; quiz 1224-5.

4. Rumboldt Z, Castillo M. Indolent intracranial mucormycosis: case report. AJNR Am J Neuroradiol. 2002; 23(6): 932-4.

5. Rosenfeld EA, Rowley AH. Infectious intracranial complications of sinusitis, other than meningitis, in children: 12-year review. Clin Infect Dis. 1994; 18(5): 750-4.

6. Juncar M, Popa AR, Lung T, Onişor F. Septic metastases of suppuration of odontogenic origin. Chirurgia (Bucur). 2011; 106(3): 359-64. [Article in Romanian]

7. Jones NS, Walker JL, Bassi S, Jones T, Punt J. The intracranial complications of rhynosinusitis: can they be prevented? Laryngoscope. 2002; 112(1): 59-63.

8. Akimura T, Ideguchi M, Kawakami N, Ito H. Brain ascess with fatal intraventricular rupture cause by asymptomatic paranasal sinusitis. Eur Arch Otorhinolaryngol. 1998; 255(7): 382. 